\title{
Glucose-Limited Fed-Batch Cultivation Strategy to Mimic Large-Scale Effects in Escherichia coli Linked to Accumulation of Non-Canonical Branched-Chain Amino Acids by Combination of Pyruvate Pulses and Dissolved Oxygen Limitation
}

\author{
Ángel Córcoles García ${ }^{1}$, Peter Hauptmann ${ }^{1}$ and Peter Neubauer ${ }^{2, *(D)}$ \\ 1 Sanofi-Aventis Deutschland GmbH, 65929 Frankfurt, Germany; angel.corcolesgarcia@sanofi.com (Á.C.G.); \\ peter.hauptmann@sanofi.com (P.H.) \\ 2 Chair of Bioprocess Engineering, Department of Biotechnology, Faculty III Process Sciences, \\ Technische Universität Berlin, 10623 Berlin, Germany \\ * Correspondence: peter.neubauer@tu-berlin.de
}

Citation: García, Á.C.; Hauptmann, P.; Neubauer, P. Glucose-Limited Fed-Batch Cultivation Strategy to Mimic Large-Scale Effects in Escherichia coli Linked to Accumulation of Non-Canonical Branched-Chain Amino Acids by Combination of Pyruvate Pulses and Dissolved Oxygen Limitation. Microorganisms 2021, 9, 1110. https://doi.org/10.3390/ microorganisms 9061110

Academic Editors: Ivana Márová and Volha Shapaval

Received: 13 March 2021

Accepted: 27 April 2021

Published: 21 May 2021

Publisher's Note: MDPI stays neutral with regard to jurisdictional claims in published maps and institutional affiliations.

Copyright: (c) 2021 by the authors. Licensee MDPI, Basel, Switzerland. This article is an open access article distributed under the terms and conditions of the Creative Commons Attribution (CC BY) license (https:/ / creativecommons.org/licenses/by/ $4.0 /)$.

\begin{abstract}
Insufficient mixing in large-scale bioreactors provokes gradient zones of substrate, dissolved oxygen (DO), $\mathrm{pH}$, and other parameters. E. coli responds to a high glucose, low oxygen feeding zone with the accumulation of mixed acid fermentation products, especially formate, but also with the synthesis of non-canonical amino acids, such as norvaline, norleucine and $\beta$-methylnorleucine. These amino acids can be mis-incorporated into recombinant products, which causes a problem for pharmaceutical production whose solution is not trivial. While these effects can also be observed in scale down bioreactor systems, these are challenging to operate. Especially the high-throughput screening of clone libraries is not easy, as fed-batch cultivations would need to be controlled via repeated glucose pulses with simultaneous oxygen limitation, as has been demonstrated in well controlled robotic systems. Here we show that not only glucose pulses in combination with oxygen limitation can provoke the synthesis of these non-canonical branched-chain amino acids (ncBCAA), but also that pyruvate pulses produce the same effect. Therefore, we combined the enzyme-based glucose delivery method Enbase ${ }^{\circledR}$ in a PALL24 mini-bioreactor system and combined repeated pyruvate pulses with simultaneous reduction of the aeration rate. These cultivation conditions produced an increase in the non-canonical branched chain amino acids norvaline and norleucine in both the intracellular soluble protein and inclusion body fractions with mini-proinsulin as an example product, and this effect was verified in a $15 \mathrm{~L}$ stirred tank bioreactor (STR). To our opinion this cultivation strategy is easy to apply for the screening of strain libraries under standard laboratory conditions if no complex robotic and well controlled parallel cultivation devices are available.
\end{abstract}

Keywords: non-canonical branched chain amino acids; scale-down; strain screening; mixed-acid fermentation; pyruvate pulse

\section{Introduction}

The scale-up of recombinant protein production processes from laboratory scale to industrial scale reactors often leads to problems, which are basically connected to the longer mixing times in large-scale bioreactors. For instance, for an E. coli-based recombinant protein production process a $20 \%$ reduction of biomass yield and an increase of by-product formation was reported when scaling up from $3 \mathrm{~L}$ to $9 \mathrm{~m}^{3}$ [1]. Furthermore, it was reported that scaling up an E. coli process from $30 \mathrm{~L}$ to $450 \mathrm{~L}$ results in biomass reduction and a lower product yield [2]. During fermentation, gradient zones of substrate, dissolved oxygen (DO), $\mathrm{pH}$ and other parameters are formed due to inefficient mixing and E. coli cells respond to these environmental changes by modulating their metabolism [3]. The standard procedure 
to grow E. coli for recombinant protein production processes in industry is the glucose limited fed-batch operation under aerobic conditions. Although it is recommended to add the glucose feed into a zone of high energy dissipation [4,5], for practical reasons, the concentrated feeding solution is often added from the top of the reactor where the local energy dissipation is low and thus aside from the longer mixing times at large scale, there are local spots or plugs with high glucose content, which are distributed only slowly. Due to the higher metabolic and respiratory activity at higher glucose concentrations, oxygen limitation occurs in the feeding zone at higher cell densities, and the increased glucose consumption in this zone leads to the exhaustion of glucose in other regions of the bioreactor. Due to the high affinity of $E$. coli to glucose-i.e., low $\mathrm{K}_{\mathrm{S}}$ (Monod substrate affinity constant) value, at higher cell densities there are nearly no zones with intermediate concentrations of glucose, i.e., cells steadily switch feast to famine conditions.

E. coli responds to glucose excess and DO limitation by shifting metabolism from oxidative respiration to mixed-acid fermentation $[5,6]$. Under oscillating conditions, the flux through the glycolysis is stimulated when the cells enter the feeding zone, resulting in high amounts of ATP and NADH as well as in the accumulation of pyruvate. This decreases the need for production of additional energy and reducing equivalents by the Krebs cycle. In this scenario, the $\mathrm{NAD}^{+}$and $\mathrm{CoA}$ pools are significantly depleted. However, the glycolytic enzyme glyceraldehyde-3-phosphate dehydrogenase requires $\mathrm{NAD}^{+}$and the pyruvate dehydrogenase complex requires $\mathrm{CoA}$ as substrate, respectively. Hence, reoxidation of NADH into $\mathrm{NAD}^{+}$and recycling of $\mathrm{CoA}$ must be ensured in order to maintain the glycolytic flux towards pyruvate and acetyl-CoA. Moreover, excess of pyruvate must be consumed. Since the Krebs cycle is inhibited, this is achieved by activating mixed-acid fermentation reactions. While acetate production serves to generate ATP, formation of other mixed-acid fermentation products regenerates $\mathrm{NAD}^{+}$and $\mathrm{CoA}$ pools [7]. Furthermore, the intracellular accumulation of pyruvate triggered by transient $\mathrm{DO}$ limitation increases the metabolic flux leading to biosynthesis of non-canonical branched-chain amino acids (ncBCAAs) through the sequential keto acid chain elongation from pyruvate to $\alpha$-ketocaproate over $\alpha$-ketobutyrate and $\alpha$-ketovalerate by the actuation of the leu operon-encoded enzymes [8]. It was proposed that a strong accumulation of pyruvate is a pre-requisite for $\alpha$-ketobutyrate formation over this shortcut since $\alpha$-isopropylmalate synthase shows lower affinity towards pyruvate than the other alternative substrates $\alpha$-ketoisovalerate and $\alpha$ ketobutyrate. Similarly, an accumulation of $\alpha$-ketobutyrate is a pre-requisite for ncBCAA biosynthesis, since the affinity of $\alpha$-isopropylmalate synthase for $\alpha$-ketobutyrate is around 20 -fold lower compared to its preferred substrate $\alpha$-ketoisovalerate [9]. NcBCAAs can be secreted to the medium and/or be mis-incorporated into native and recombinant proteins through tRNA mis-aminoacylation during protein translation. This occurs due to the promiscuity of aminoacyl-tRNA synthetases, which might accept the non-canonical amino acids instead of the canonical counterparts due to their chemical similarity (reviewed in [10]). For instance, when norvaline is present, leucyl-tRNA synthetase might react with norvaline instead of the canonical leucine. Such mis-incorporation in recombinant proteins can lead to the production of altered protein variants, having non optimal characteristics.

Scale-down bioreactors have been used in bioprocess development in order to better understand the physiological response of E.coli cells to concentration gradients occurring in industrial-scale bioreactors due to inefficient mixing. Numerous scale-down models have been developed, including one-reactor and multi-compartment reactor systems [11]. These scale-down simulators can generate feeding and/or oxygen oscillations in order to mimic scale-up effects. For instance, an accumulation of pyruvate-based amino acids such as the ncBCAAs norleucine and norvaline as well as alanine and valine was reported in a standard stirred tank reactor (STR) fed-batch $E$. coli cultivation under glucose excess and induced DO limitation upon a stirrer downshift [6]. Furthermore, in a recombinant $E$. coli cultivation, the combination of DO limitation and high glucose concentration in the feed compartment of a two-compartment stirred tank-plug flow reactor (STR-PFR) system resulted in a significantly higher norvaline biosynthesis due to pyruvate accumulation [12]. 
Although these scale-down approaches provide insights into microbial cell responses that are relevant under large-scale conditions, the complexity of the reactor systems and their laborious operation are not suitable for screening experiments. A solution to this was recently presented by Anane et al. [13], in which pulse-based scale-down experiments were performed in parallel mini-bioreactors on a high performance robotic station. The authors were able to demonstrate both the accumulation of norvaline in the cytosol and its incorporation into recombinant mini-proinsulin. However, such a high-end system is very complex to operate and thus not accessible in standard laboratories for screening purposes.

Additionally, the glucose based pulse feeding causes complex effects, connected to (i) the dependence of glucose uptake through the phosphoenolpyruvate -dependent phosphotransferase system (PTS) by the ratio of phosphoenolpyruvate to pyruvate, (ii) different uptake systems which are relevant for the glucose uptake and differently regulated in connection with growth limitation, and (iii) the branching of the glucose flux in the upper glycolytic pathway, e.g., to the pentose phosphate shunt. Therefore, we developed the idea to apply pyruvate pulses. Pyruvate is the core substrate for both mixed-acid fermentation and branched chain amino acid biosynthetic reactions. Both pathways are activated in large-scale reactors. In addition, unlike glucose, pyruvate is transported into the cell through the PrvT and Usp transporters and thus independent from the PTS [14]. Furthermore, its uptake and metabolism is not dependent on the energy status of the cell. In our approach the pyruvate pulses are applied in cultivations which were performed as glucose-limited fed-batch cultures with the enzyme-based glucose delivery strategy (Enbase $\left.{ }^{\circledR},[15]\right)$. These cultivations provide conditions which are relevant to industrial operation and additionally can be very easily set up for high throughput screenings.

\section{Materials and Methods}

\subsection{Strain, Plasmid, and Cultivation Medium}

E. coli K-12 BW25113 containing the plasmid pSW3_lacI ${ }^{+}$(Figure S1) was used in this study. This plasmid expresses a recombinant mini-proinsulin (MPI) under the control of an isopropyl- $\beta$-D-thiogalactopyranosid (IPTG)-inducible tac-promoter. The cultivations were performed in mineral salt medium containing (per L): $2.0 \mathrm{~g} \mathrm{Na}_{2} \mathrm{SO}_{4}, 2.468 \mathrm{~g}\left(\mathrm{NH}_{4}\right)_{2} \mathrm{SO}_{4}$, $0.5 \mathrm{~g} \mathrm{NH}_{4} \mathrm{Cl}, 14.6 \mathrm{~g} \mathrm{~K}_{2} \mathrm{HPO}_{4}, 3.6 \mathrm{~g} \mathrm{NaH}_{2} \mathrm{PO}_{4} \times 2 \mathrm{H}_{2} \mathrm{O}$ and $1.0 \mathrm{~g}\left(\mathrm{NH}_{4}\right)_{2}-\mathrm{H}$-citrate, $2 \mathrm{~mL}$ of a $1.0 \mathrm{M} \mathrm{MgSO}_{4}$ solution, and $2 \mathrm{~mL}$ of a trace elements solution. The trace elements solution contained (per L): $0.5 \mathrm{~g} \mathrm{CaCl}_{2} \times 2 \mathrm{H}_{2} \mathrm{O}, 0.18 \mathrm{~g} \mathrm{ZnSO}_{4} \times 7 \mathrm{H}_{2} \mathrm{O}, 0.1 \mathrm{~g} \mathrm{MnSO}_{4} \times \mathrm{H}_{2} \mathrm{O}, 16.7 \mathrm{~g}$ $\mathrm{FeCl}_{3} \times 6 \mathrm{H}_{2} \mathrm{O}, 0.16 \mathrm{~g} \mathrm{CuSO}_{4} \times 5 \mathrm{H}_{2} \mathrm{O}$ and $0.18 \mathrm{~g} \mathrm{CoCl}_{2} \times 6 \mathrm{H}_{2} \mathrm{O}$. Additionally, the medium contained the carbon source as described below. Most of the aforementioned chemicals were acquired from Sigma-Aldrich (Munich, Germany), with exception of $\mathrm{NH}_{4} \mathrm{Cl}$ and $\mathrm{MnSO}_{4} \times \mathrm{H}_{2} \mathrm{O}$, which were acquired from Merck (Darmstadt, Germany).

\subsection{Cultivation Conditions in a Mini-Reactor System}

In this study, two different cultivation modes were tested in a Pall Micro24 reactor system (Microreactor Technologies Inc., Mountain View, CA, USA). The first mode is a reference cultivation consisting of a glucose-limited fed-batch cultivation under aerobic conditions. The second mode is the same kind of glucose-limited fed-batch cultivation but additionally pyruvate pulses and transient down-shifts of the oxygen supply were applied in order to trigger $\mathrm{DO}$ limitation.

As pre-culture for the mini-bioreactor cultivations $30 \mu \mathrm{L}$ of a cryostock containing $E$. coli BW25113 pSW3_lac ${ }^{+}$were used to inoculate a $250 \mathrm{~mL}$ Erlenmeyer flask with $30 \mathrm{~mL}$ of 1:3 diluted supplemented mineral salt medium containing $5 \mathrm{~g} \mathrm{~L}^{-1}$ glucose (Merck, Darmstadt, Germany), 0.1 M Na-phosphate buffer (Merck, Darmstadt, Germany) and $100 \mu \mathrm{g} \mathrm{mL}^{-1}$ ampicillin (Sigma-Aldrich, Munich, Germany). The pre-culture was incubated at $37{ }^{\circ} \mathrm{C}$ and $220 \mathrm{rpm}$ in the orbital ISF1-X shaker (Adolf Kühner AG, Birsfelden, Switzerland), overnight. $\mathrm{OD}_{600}$ at the end of the pre-culture was measured and a given volume was used to inoculate the mini-bioreactors Pall Micro24 with a total starting volume of $5 \mathrm{~mL}$ so that initial $\mathrm{OD}_{600}$ was 0.4 . The mini-reactor medium consisted of a 1:3 diluted 
supplemented mineral salt medium containing $4 \mathrm{~g} \mathrm{~L}^{-1}$ glucose, $0.1 \mathrm{M} \mathrm{Na}$-phosphate buffer, $100 \mu \mathrm{g} \mathrm{mL}^{-1}$ ampicillin and $1 \mu \mathrm{L} \mathrm{mL}^{-1}$ antifoam Desmophen (Covestro AG, Leverkusen, Germany). For the minibioreactors the 1:3 diluted medium had to be used instead of a standard mineral salt medium, which had been used in the $15 \mathrm{~L}$ bioreactors, because we observed salt precipitation which was clogging the oxygen vent. Cultivations were performed at $37^{\circ} \mathrm{C}$ and the $\mathrm{pH}$ was maintained at 7.0 by automatic control with $\mathrm{NH}_{4} \mathrm{OH}$ (Bernd Kraft GmbH, Duisburg, Germany) and $\mathrm{CO}_{2}$. Stirrer speed was set to $800 \mathrm{rpm}$ and DO set-point to $25 \%$, maintaining the last by automatically increasing the oxygen flow into the mini-reactor. After the end of the batch phase (approx. $4 \mathrm{~h}$ ) $1 \mathrm{~mL}$ of a $400 \mathrm{~g} \mathrm{~L}^{-1}$ EnPump 200 solution and $50 \mu \mathrm{L}$ of a $3000 \mathrm{U} \mathrm{L}^{-1}$ glucoamylase solution (Enpresso $\mathrm{GmbH}$, Berlin, Germany) were manually added into each of the mini-reactors, hence starting the fed-batch phase. EnPump 200 is a soluble starch-derived glucose polymer which is hydrolyzed by glucoamylase and thus delivers free glucose molecules over time, ensuring a glucose-limited cultivation mode. In order to generate the $400 \mathrm{~g} \mathrm{~L}^{-1}$ EnPump 200 solution, $25 \mathrm{~g}$ EnPump 200 (Enpresso GmbH, Berlin, Germany) powder was dissolved in a $25 \mathrm{~mL}$ diluted mineral salt medium, so that components' concentration in the mini-reactor remained invariable after adding the EnPump $30 \mathrm{~min}$ after beginning of the fed-batch phase. The recombinant mini-proinsulin was induced by a single pulse of IPTG (Sigma-Aldrich, Munich, Germany) to a final concentration of $0.5 \mathrm{mM}$. The fed-batch phase was further maintained for $3.5 \mathrm{~h}$.

For the cultivation with pyruvate pulses and DO limitation, immediately after beginning of the fed-batch phase, a $0.833 \mathrm{~g} \mathrm{~L}^{-1}$ pyruvate (Sigma-Aldrich, Munich, Germany) pulse was manually added into the reactor. During the following $5 \mathrm{~min}$ after pyruvate addition, DO set-point was set to 0 , so that no oxygen was supplied into the mini-reactor during that period. $30 \mathrm{~min}$ after the first pyruvate pulse, expression of recombinant miniproinsulin was induced by IPTG as described above. After induction, repeated $0.833 \mathrm{~g} \mathrm{~L}^{-1}$ pyruvate pulses were manually performed each $30 \mathrm{~min}$ for a total of 5 pulses. Between pulses, DO set-point was re-established to $25 \%$.

\subsection{Cultivation Conditions in the 15 L Stirred-Tank Reactor}

As for the mini-reactor system, two different cultivation modes were tested in a $15 \mathrm{~L}$ stirred-tank reactor.

In order to generate the pre-culture of the reference cultivation $100 \mu \mathrm{L}$ of a cryostock of E. coli K-12 BW25113 pSW3_lacI ${ }^{+}$were used to inoculate an Erlenmeyer flask with $500 \mathrm{~mL}$ of supplemented mineral salt medium containing $5 \mathrm{~g} \mathrm{~L}^{-1}$ glucose and $100 \mu \mathrm{g} \mathrm{mL} \mathrm{L}^{-1}$ ampicillin. The pre-culture was incubated at $37^{\circ} \mathrm{C}$ and $220 \mathrm{rpm}$ in an orbital shaker for $12 \mathrm{~h} . \mathrm{OD}_{600}$ at the end of the pre-culture was measured and a given volume was used to inoculate the bioreactor (Type 880142.8, Nr. 209, Braun Melsungen AG, Melsungen, Germany) with 7 L starting volume reactor so that the initial $\mathrm{OD}_{600}$ was 0.4 . The reactor medium consisted of mineral salt medium with $5 \mathrm{~g} \mathrm{~L}^{-1}$ glucose, $2 \mathrm{~mL}$ antifoam 204 (Sigma-Aldrich, Munich, Germany) and $100 \mathrm{mg} \mathrm{L}^{-1}$ ampicillin. Cultivation was carried out at $37^{\circ} \mathrm{C}$ and the $\mathrm{pH}$ was maintained at 7.0 by automatic control with $25 \% \mathrm{NH}_{4} \mathrm{OH}$. Airflow was set to $7 \mathrm{vvm}$ and DO set-point to $20 \%$, by using a cascade control of the stirrer speed (initial stirrer speed was $800 \mathrm{rpm}$ ). Exponential feed was started after the end of the batch phase (approx. $4 \mathrm{~h}$ ) according to following equation,

$$
F(t)=\frac{q_{s}}{S_{i}} \cdot\left(X_{0} \cdot V_{0}\right) \cdot e^{\mu_{s e t} \cdot t}
$$

where $F(t)$ represents the feed rate over time $\left(\mathrm{L} \mathrm{h}^{-1}\right), q_{s}$ the set-point of the specific glucose uptake rate $\left(0.514 \mathrm{~g} \mathrm{~g}^{-1} \mathrm{~h}^{-1}\right), \mathrm{S}_{\mathrm{i}}$ the concentration of glucose in the feed solution $\left(442 \mathrm{~g} \mathrm{~L}^{-1}\right)$, $X_{0}$ the biomass concentration at the feed start $\left(\mathrm{g} \mathrm{L}^{-1}\right), V_{0}$ the volume of the reactor at the feed start (L), $\mu_{\text {set }}$ the set-point of the specific cell growth rate $\left(0.3 \mathrm{~h}^{-1}\right)$ and $t$ the time after feed start. The feed solution consisted of mineral salt medium supplemented with $4 \mathrm{~mL} \mathrm{~L}^{-1}$ trace elements solution, $2 \mathrm{~mL} \mathrm{~L}^{-1} \mathrm{MgSO}_{4}$ solution $(1.0 \mathrm{M}), 100 \mathrm{mg} \mathrm{L}^{-1}$ ampicillin and 
$442 \mathrm{~g} \mathrm{~L}^{-1}$ glucose. The exponential feed was continued for $3 \mathrm{~h}$ and afterwards expression of recombinant mini-proinsulin was induced by automatic addition of $0.5 \mathrm{mM}$ IPTG over a time period of $30 \mathrm{~min}$. During this time no feed was added into the reactor. After induction, the feed was re-started with a constant flow rate which was equal to the last flow rate achieved in the exponential feeding phase. This constant feed fed-batch phase was maintained for $6 \mathrm{~h}$. A general overview of the cultivation is shown in Figure S2.

The cultivation exposed to pyruvate pulses and DO limitation was performed similar, but after the exponential fed-batch phase a pyruvate solution was added by the feed pump for $5 \mathrm{~min}$ into the reactor, providing a final amount of approx. $1 \mathrm{~g} \mathrm{~L}^{-1}$ pyruvate. During this time no glucose feed was added, the airflow rate was temporary set to 0 and the DO cascade control was stopped, so that the DO decreased to zero. Directly after the pyruvate pulse, expression of recombinant mini-proinsulin was induced by automatic addition of $0.5 \mathrm{mM}$ IPTG over $30 \mathrm{~min}$, as described above. During IPTG addition no glucose feed was added and airflow and DO cascade control were re-established to the setpoints before the pyruvate pulse. After induction, successive $1 \mathrm{~g} \mathrm{~L}^{-1}$ pyruvate pulses were applied by $5 \mathrm{~min}$ feed intervals every $30 \mathrm{~min}$ as described above, for a total of 4 pulses. Between pulses, the constant glucose feeding phase was activated, so that the constant flow rate was equal to the last flow rate achieved in the exponential feeding phase, and airflow and DO cascade control were re-established. Here the constant feed fed-batch phase was continued for $5 \mathrm{~h}$. A general overview of the cultivation is shown in Figure S3.

\subsection{Cell Growth and Mini-Proinsulin Analysis}

Cell growth was monitored by measuring the optical density at a wavelength of $600 \mathrm{~nm}\left(\mathrm{OD}_{600}\right)$ in a photometer Ultraspec 2100 pro (Amersham Bioscience, Marlborough, MA, USA). When applicable, samples were diluted with the original medium into an $\mathrm{OD}_{600}$ range of 0.3-0.8. Concentration of recombinant mini-proinsulin from hourly samples taken from the cultivations carried out in the STR was analyzed according to an HPLC method internally available at Sanofi-Aventis Deutschland GmbH.

\subsection{Acetate and Formate Analysis}

Acetate and formate concentrations from STR cultivation samples were analyzed offline by enzymatic assays internally available at Sanofi-Aventis Deutschland GmbH.

\subsection{NcBCAA Analysis}

Isolation of the intracellular soluble protein and inclusion body fractions from total cell extracts was carried out as described in the protocol available for BugBuster Protein Extraction Reagent (Merck, Darmstadt, Germany). A certain volume (max. $250 \mu \mathrm{L}$ ) of the isolated protein fractions was mixed up to $1 \mathrm{~mL}$ with $5 \mathrm{M} \mathrm{HCl}$ (Sigma-Aldrich, Munich, Germany). Resulting solutions were introduced in crystal vials with screw caps resistant to aggressive acids. Closed vials were incubated for $24 \mathrm{~h}$ at $80^{\circ} \mathrm{C}$ for acid hydrolysis of the cellular proteins. Afterwards, vials were left open in a heating block for 16 to $24 \mathrm{~h}$ at $65^{\circ} \mathrm{C}$ while rotating until all liquid was evaporated. Hydrolyzed samples were then resuspended with a solution containing $20 \mathrm{mM} \mathrm{HCl}$ and $10 \%$ isopropanol (Acros Organics ThermoFisher Scientific, Waltham, MA, USA). Resulting solutions were used for further amino acid isolation according to the protocol provided by the EZ:faast ${ }^{\mathrm{TM}}$ kit for free (physiological) amino acids by GC-FID (Phenomenex, Aschaffenburg, Germany) with $\alpha$-aminobutyric acid (Sigma-Aldrich, Munich, Germany) as internal standard. After amino acid isolation, approximately $120 \mu \mathrm{L}$ of the resulting upper layer were introduced into GC vials and $2 \mu \mathrm{L}$ were then injected into the GC-FID analyzer 7890A (Agilent Technologies, Santa Clara, CA, USA). Concentration working range was $1-200 \mu \mathrm{M}$. The GC was run according to following oven conditions: equilibration time of $0.5 \mathrm{~min}, 110{ }^{\circ} \mathrm{C}$ for $1 \mathrm{~min}, 30^{\circ} \mathrm{C} \mathrm{min}-1$ heating up to $320^{\circ} \mathrm{C}$ and then $320^{\circ} \mathrm{C}$ for $1 \mathrm{~min}$. Each GC run was about $9 \mathrm{~min}$. Nitrogen was used as a carrier gas, with a constant flow rate of $1.5 \mathrm{~mL} \mathrm{~min}^{-1}$. Injection was carried out with a $1: 15$ split ratio at $250^{\circ} \mathrm{C}$. 
NcBCAA concentrations were determined from hourly samples taken from cultivations carried out in the STR. From the cultivations in the mini-reactor system ncBCAA concentrations were just determined at the end of the cultivation ( $3 \mathrm{~h}$ after induction) due to the limited available volumes.

\section{Results}

Effect of Pyruvate Pulsing and DO Limitation on ncBCAA Biosynthesis and Mis-Incorporation into Recombinant Mini-Proinsulin

It is known that the biosynthesis and mis-incorporation of ncBCAAs into recombinant proteins occurs during $E$. coli based recombinant protein production processes with perturbations in the glucose and dissolved oxygen (see [13]). In this study, we postulate that the repeated application of pyruvate pulses with simultaneous DO limitation in a fed-batch background triggers the synthesis of ncBCAAs in a similar way. Hence, in order to verify our hypothesis, we performed glucose limited fed-batch cultivations with or without pyruvate pulses and analyzed the levels of the ncBCAAs in both intracellular soluble protein and inclusion body fractions of a mini-proinsulin. While the pyruvate pulses with the concomitant $\mathrm{DO}$ limitation had no obvious effect on neither the $\mathrm{OD}_{600}$ nor on the accumulation of the mini-proinsulin (Figure 1), the concentrations of all three ncBCAAs showed an increase in the intracellular soluble protein fraction after induction of mini-proinsulin production in the cultivations with the pyruvate pulses and concomitant DO limitation (Figure 2). While the concentrations of all of them were similar before induction, norvaline and norleucine showed a stronger increase and higher concentrations than $\beta$-methylnorleucine. While the intracellular concentration of all three ncBCAAs increased also in the reference cultivations, the accumulation was much higher in the pulsed cultivations, especially for norvaline and norleucine, while there was only a minor difference in the levels of $\beta$-methylnorleucine. Interestingly, while the concentrations of norleucine and $\beta$-methylnorleucine increased all the time, norvaline had a maximum level $3 \mathrm{~h}$ after induction and dropped then.

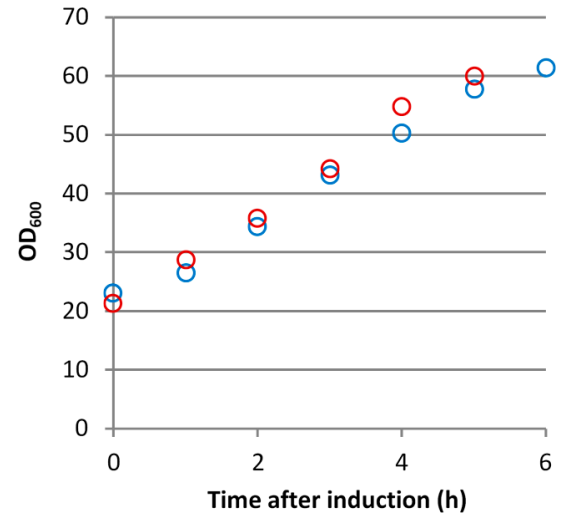

(a)

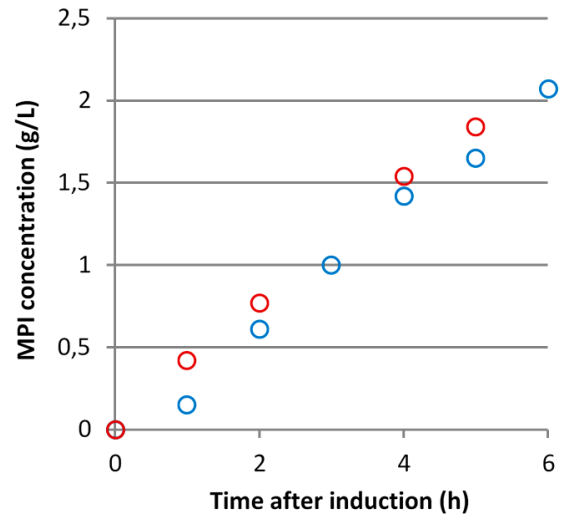

(b)

Figure 1. Measured $\mathrm{OD}_{600}$ (a) and concentrations of recombinant mini-proinsulin (MPI) (b) over time after induction of E. coli BW25113 pSW3_lacI ${ }^{+}$in a fed-batch cultivation in a 15 L bioreactor under reference cultivation conditions (blue symbols) or with pyruvate pulsing and concomitant dissolved oxygen (DO) limitation (red symbols). Results correspond to the average of three technical replicates. The first measured sample corresponds to the time-point right before induction. 


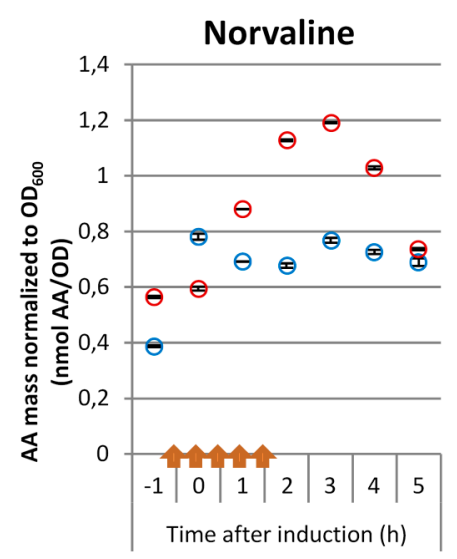

(a)

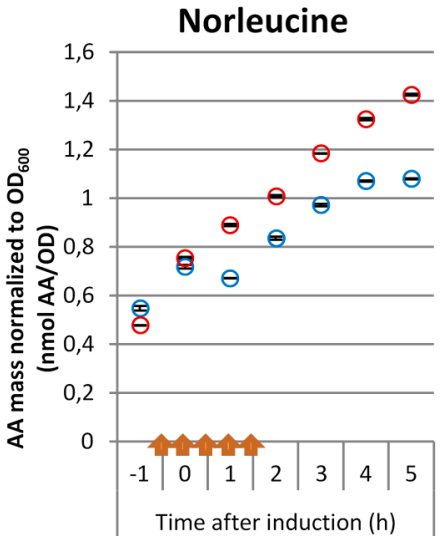

(b)

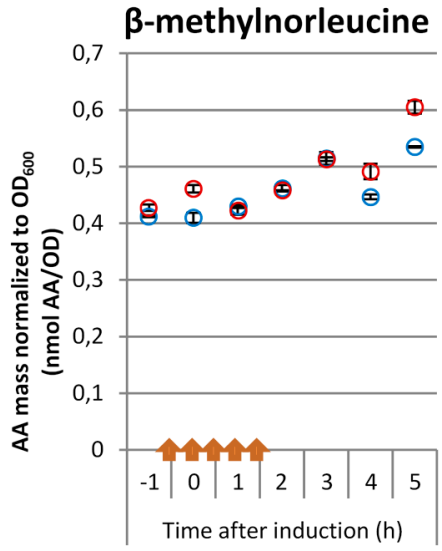

(c)

Figure 2. Molar concentrations of norvaline (a), norleucine (b) and $\beta$-methylnorleucine (c) normalized to $\mathrm{OD}_{600}$ in the intracellular soluble fraction after induction of E. coli BW25113 pSW3_lacI ${ }^{+}$glucose limited fed-batch cultivation in a $15 \mathrm{~L}$ stirred tank reactor (STR) under reference cultivation conditions (blue symbols) or in a cultivation with pyruvate pulses and concomitant dissolved oxygen (DO) limitation (red symbols). Arrows indicate time points where $1 \mathrm{~g} \mathrm{~L}^{-1}$ pyruvate pulse combined with 5 min DO limitation was applied. Results correspond to the average of 3 technical replicates.

For both tested cultivation types the accumulation of norvaline and norleucine was also observed in the inclusion body fraction (Figure 3), while the concentration of $\beta$ methylnorleucine in the inclusion body fraction was below the detection limit. In the inclusion body fraction the accumulation of norleucine was nearly 10-fold higher than that of norvaline, although it was less dependent on the pulses, while the norvaline accumulation in the inclusion body fraction was much higher in the pulsed cultivation compared to the reference. This difference became especially obvious from $3 \mathrm{~h}$ after induction onwards, i.e., in the period when the intracellular concentration of norvaline decreased.

As the synthesis of the ncBCAAs is considered as an overflow metabolic reaction from pyruvate, it also was interesting to see the response of mixed-acid fermentation products in these cultivations. For both tested cultivation conditions, the extracellular concentration of acetate was highest at the end of the batch phase (Figure 4a). As expected, it decreased in the first phase of the fed-batch cultivation. A second increase of acetate was observed after induction, while the increase due to the intermittent pyruvate pulses with concomitant DO limitation was not very significant. The difference in acetate concentrations between the pulsed cultivations and the reference becomes only obvious approx. $2 \mathrm{~h}$ after induction. The sudden and momentary accumulation of acetate during the fed-batch phase in the reference cultivation corresponds with a sudden glucose accumulation after induction due to the relatively high feeding rate applied (Figure S2). 


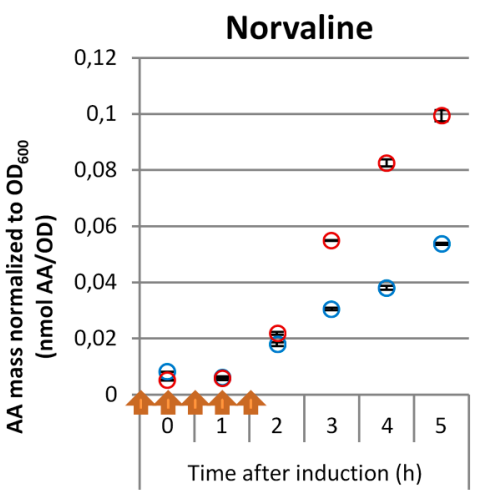

(a)

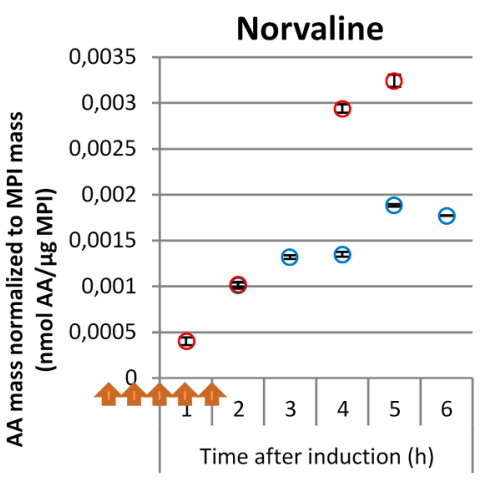

(c)

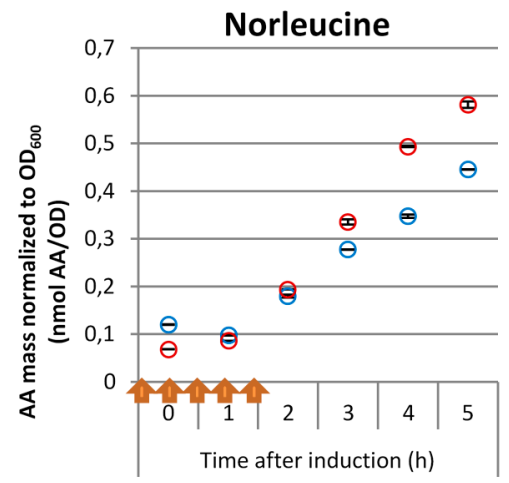

(b)

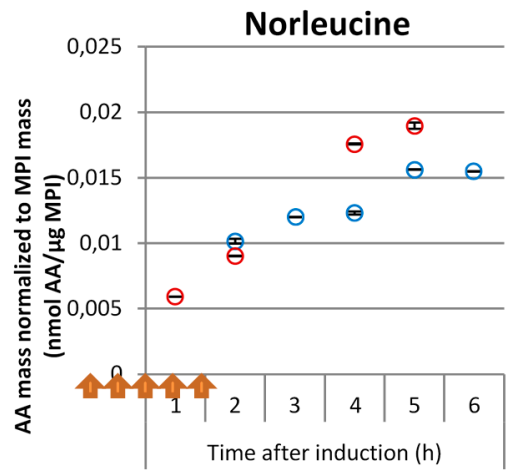

(d)

Figure 3. Molar concentrations of norvaline $(\mathbf{a}, \mathbf{c})$ and norleucine $(\mathbf{b}, \mathbf{d})$ normalized to $\mathrm{OD}_{600}(\mathbf{a}, \mathbf{b})$ or to recombinant mini-proinsulin (MPI) mass $(\mathbf{c}, \mathbf{d})$ in the inclusion body fraction after induction of $E$. coli BW25113 pSW3_lacI ${ }^{+}$glucose limited fed-batch cultivation in a 15 L stirred tank reactor (STR) under reference cultivation conditions (blue symbols) or in a cultivation with pyruvate pulses and concomitant DO limitation (red symbols). Arrows indicate time points of $1 \mathrm{~g} \mathrm{~L}^{-1}$ pyruvate pulses combined with $5 \mathrm{~min}$ DO limitation. Results correspond to the average of 3 technical replicates.

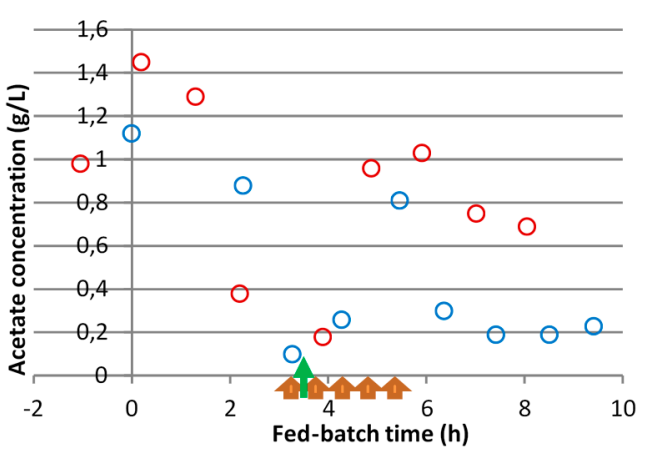

(a)

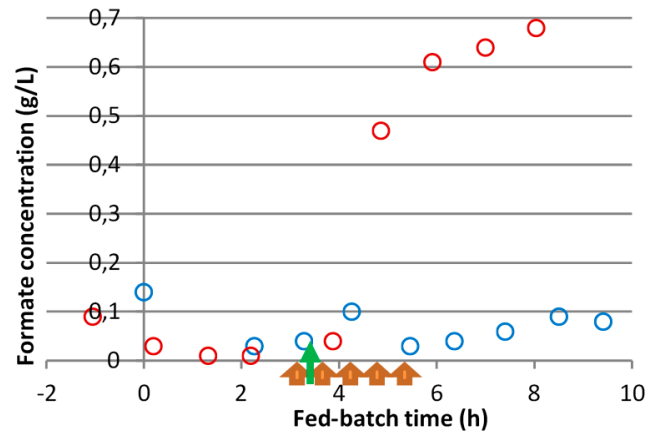

(b)

Figure 4. Concentration of acetate (a) and formate (b) in culture broth supernatants during the

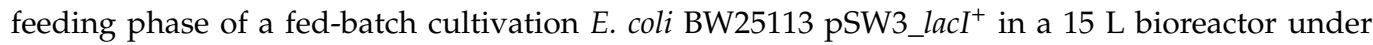
reference cultivation conditions (blue symbols) or under concomitant pyruvate pulsing and dissolved oxygen (DO) limitation (red symbols), respectively. Orange arrows indicate times of $1 \mathrm{~g} \mathrm{~L}^{-1}$ pyruvate pulses each combined with $5 \mathrm{~min}$ DO limitation. Green arrow shows the time of IPTG induction. Results represent the average of 3 technical replicates.

Unlike acetate, formate is known to accumulate only in response to oxygen limitation. Therefore, as expected, its concentration was low at the end of the batch phase and it disappeared in the first phase of the fed-batch cultivation (Figure $4 \mathrm{~b}$ ). While induction only caused a very slight transient increase of formate, the pyruvate pulses together with the DO limitation caused a very strong increase of formate. As has been previously discussed 
formate is not easily re-metabolized in standard mineral salt media $[16,17]$ and it thus simply accumulates as an indicator of oxygen limitation.

As we believe that the use of pyruvate pulses could be valuable for screening of clone libraries, we applied it in a simple mini-bioreactor system, the Pall Micro24 bioreactor. This reactor is a stand-alone $10 \mathrm{~mL}$ cultivation system based on 24 bubble columns. While it offers the advantage of performing parallel cultivation, it has no manual continuous feeding option and also sampling is limited. Therefore, in these cultivations we applied the Enbase enzyme-based glucose delivery system, which imitates a glucose limited fed-batch, and we analyzed the inclusion body and intracellular soluble protein fractions at the end of the cultivations. To obtain validated results three biological replicates were performed, i.e., different cultures were performed for each type of cultivation (Figure 5). Pyruvate pulses in combination with DO limitation, which was realized by a sudden decrease of the aeration rate, in these mini-bioreactor experiments also lead to an increase of norvaline by $17.5 \%$ $(\mathrm{t}=1.14, p=0.32)$, and norleucine by $51.7 \%(\mathrm{t}=3.21, p=0.03)$, while $\beta$-methylnorleucine decreased by $14.8 \%(\mathrm{t}=2.82, p=0.05)$ in the intracellular soluble protein fraction. In the inclusion body fraction norvaline increased by $25.6 \%(t=0.57, p=0.61)$ and norleucine by $28.1 \%(t=1.29, p=0.27)$. Although the increase is statistically significant only for norleucine in the intracellular protein fraction, the trend for all of these ncBCAAs is similar to the $15 \mathrm{~L}$ bioreactor cultivation and also similar to our previous data of the analysis of the free ncBCAAs during production of the same mini-proinsulin where the increase of the level of norleucine was most prominent, while the concentration of norvaline was only about one third of it, and the increase of $\beta$-methylnorleucine was lowest [18]. However, it is interesting and deserves further investigations that the level of $\beta$-methylnorleucine was much higher in these small-scale cultivations compared to the $15 \mathrm{~L}$ bioreactor.

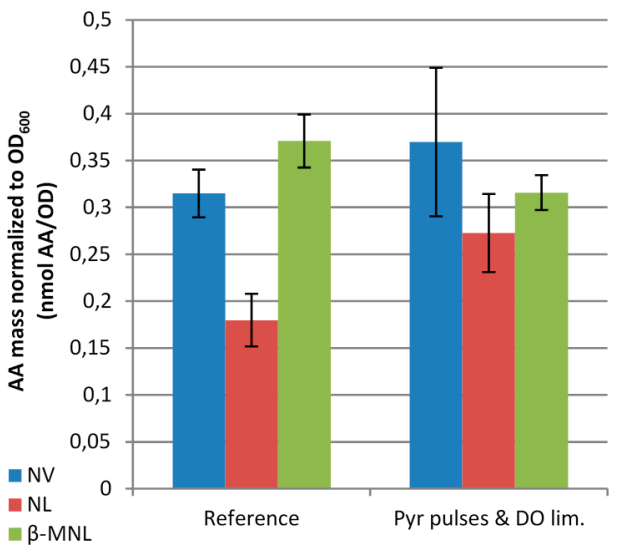

(a)

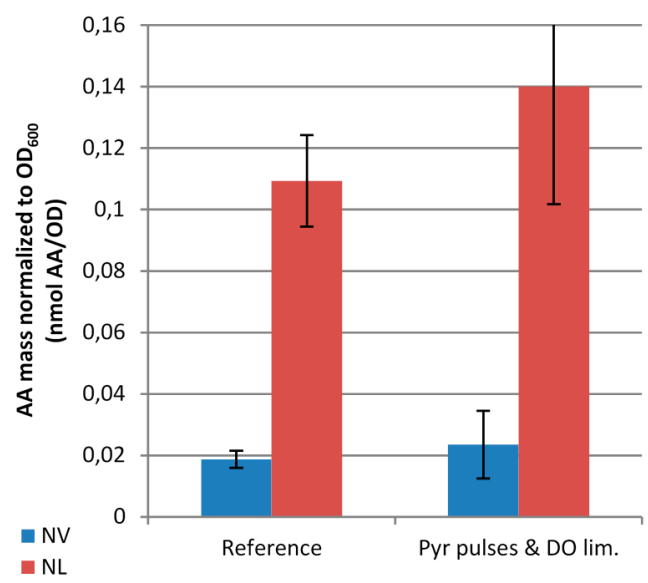

(b)

Figure 5. Molar concentrations of norvaline (NV), norleucine (NL) and $\beta$-methylnorleucine ( $\beta$ MNL) normalized to $\mathrm{OD}_{600}$ in the hydrolyzed intracellular soluble protein fraction (a) and inclusion body fraction (b) at $3 \mathrm{~h}$ after induction of E. coli BW25113 pSW3_lacI ${ }^{+}$cultivation in $10 \mathrm{~mL}$ minibioreactor cultivations under reference conditions (Reference) or under simultaneous pyruvate pulsing and dissolved oxygen (DO) limitation (Pyr pulses and DO lim.). Results represent the average of 3 independent biological replicates.

\section{Discussion}

Here we demonstrated that the combination of pyruvate pulses and dissolved oxygen limitation in a fed-batch background can be used as an alternative strategy to simulate the effects of a transient pyruvate accumulation which results in the accumulation of non-canonical branched chain amino acids. This in turn can lead to the mis-incorporation of these amino acids into recombinant proteins and thus to quality problems, especially in pharmaceutical production. We believe that the use of pyruvate compared to glucose bolus addition can have significant advantages as pyruvate is the direct starting point of 
the metabolic pathway leading to the synthesis of the ncBCAAs. Hence, when adding pyruvate to the cultivation medium, this enters the cell and accumulates intracellularly, rapidly triggering ncBCAA formation through the sequential keto acid chain elongation by the actuation of the enzymes encoded by the leu operon.

Unlike pyruvate, when using glucose pulses, the metabolic pathway is not only longer but also much more regulated. The standard glucose uptake system in E. coli is the glucose phosphoenolpyruvate: carbohydrate phosphotransferase system (glucose PTS). In this system the actual glucose uptake rate is directly dependent on the ratio of phosphoenolpyruvate to pyruvate which changes fast during a glucose pulse. This ratio depends not only on the regulation of this uptake system itself and the flux through the upper part of the glycolysis, but also from the flux of pyruvate to acetyl CoA either by pyruvate dehydrogenase or, alternatively under oxygen limitation, over the pyruvate formate lyase aside from other metabolic reactions which include pyruvate. Furthermore, aside from the glucose PTS, glucose is also assimilated by many other transporters, such as the mannose PTS system, the galactose $\mathrm{ABC}$ transporter, the galactose permease, and the maltose $\mathrm{ABC}$ transporter [19].

Also, pyruvate is taken up by different transporters. Kreth et al. [14] discussed the existence of at least three transport systems for pyruvate in order to equilibrate intracellular pyruvate concentrations: an inducible uptake system (Usp system), a constitutive uptake system (PrvT system) and an excretion system. The first two systems were demonstrated to be controlled by catabolite repression. Under cultivation conditions subjected to glucose limitation, pyruvate can be then easily transported into the cell through the PrvT and Usp transport systems.

Thus, if pyruvate pulses are used, it is not only closer to the ncBCAA route, but also the intracellular pyruvate concentration is regulated by the transporters which should result in more robust system which is less dependent on other environmental conditions if the experiments are performed under glucose limitation.

In praxis so far, a shift from aerobic conditions to oxygen limitation at either high glucose concentration [6] or in combination with a glucose bolus addition have succeeded in the best production of the ncBCAAs. The latter procedure was applied during investigations which aimed at imitating large scale industrial cultivations in a laboratory environment, i.e., in a scale down simulator [13]. Generally, the time of accumulation of the ncBCAAs was similar in the glucose shift studies and here; it took about one to $1.5 \mathrm{~h}$ to see significant changes.

Mis-incorporation of ncBCAAs during translation can lead to the production of altered proteins, having non optimal characteristics. There is plenty of evidence showing that the mis-incorporation of norleucine into recombinant proteins by E. coli may lead to alteration of protein structure and biological properties. For instance, recombinant norleucine-substituted $\beta$-galactosidase was more resistant to alkylation [20] and recombinant norleucine-rich mammalian calmodulin reported a decreased enzymatic activity in comparison with the canonical variant [21]. When recombinant proteins are to be used as pharmaceutical products for human use, a number of quality criteria have to be fulfilled in order to ensure its effectiveness and safety when delivered in the market. Hence, a number of product parameters have to be tested and these should meet the specifications. One of those parameters is the purity level of the product. The mis-incorporation of ncBCAAs into the product during the recombinant protein production process generates a pool of protein variants differing by only a few amino acids. During downstream processing these variants cannot be easily removed since most protein variants show very similar properties as the main product. Therefore, this issue represents an important concern for the pharmaceutical industry [22]. Also, strategies to suppress mis-incorporation of the no-canonical amino acids by process parameters, such as feeding of leucine or avoiding glucose gradients are not straight forward, especially in industrial large-scale reactors [10]. Hence, the selection of $E$. coli strains showing a better product purity profile is crucial prior to scale-up. However, such screenings have not been extensively applied so far due to 
methodological limitations. This is where the here proposed method could be applied, which was shown also to function in a simple mini-bioreactor.

\section{Conclusions}

The cultivation strategy based on the combination of pyruvate pulses and dissolved oxygen limitation in a fed-batch background represents a novel approach to mimic the conditions which lead to the accumulation of ncBCAA and this could be shown in a standard benchtop bioreactor and in a simple parallel mini bioreactor system. We believe the system with pyruvate pulses is so easy that it can be used for the analysis of the ncBCAA potential of whole clone libraries. Such a robustness analysis would be highly important for the development of new large-scale processes of recombinant proteins, to avoid misincorporation issues from the beginning. With such a parallel cultivation procedure based on fed-batch conditions in a batch system, bacterial strains showing a better product purity profile, i.e., reduced ncBCAA mis-incorporation, could be selected, thus ensuring a higher product quality.

Supplementary Materials: The following are available online at https:/ /www.mdpi.com/article/10 $.3390 /$ microorganisms $9061110 /$ s1. Figure S1. Genetic map of plasmid pSW3_lacI ${ }^{+}$. Plasmid map was generated by Snapgene ${ }^{\circledR}$. Figure S2. Overview of the reference cultivation of E. coli K-12 BW25113 pSW3_lacI ${ }^{+}$in a 15L reactor, during the whole cultivation process (a) and during fed-batch period (b). Figure S3. Overview of the cultivation of E. coli K-12 BW25113 pSW3_lacI ${ }^{+}$exposed to pyruvate pulses and DO limitation in a $15 \mathrm{~L}$ reactor, during the whole cultivation process (a), during fed-batch period (b) and during pyruvate pulsing (c).

Author Contributions: Á.C.G. has performed the experiments, interpreted the results, and drafted the manuscript. P.H. and P.N. both supported experimental design, interpreted the results, and reviewed the manuscript. All authors have read and agreed to the published version of the manuscript.

Funding: This research was funded and performed by the European Union's Horizon2020 research and innovation program under the Marie Skłodowska-Curie actions grant agreement No. 643056 (Biorapid). This research is part of the dissertation by Á.C.G. published 2020 by the library of the TU Berlin (Germany) (10.14279/depositonce-10650).

Institutional Review Board Statement: Not applicable.

Informed Consent Statement: Not applicable.

Conflicts of Interest: The authors declare no conflict of interest. The funders had no role in the design of the study; in the collection, analyses, or interpretation of data; in the writing of the manuscript, or in the decision to publish the results.

\section{References}

1. Bylund, F.; Collet, E.; Enfors, S.O.; Larsson, G. Substrate gradient formation in the large-scale bioreactor lowers cell yield and increases by-product formation. Bioprocess Eng. 1998, 18, 171-180. [CrossRef]

2. Riesenberg, D.; Menzel, K.; Schulz, V.; Schumann, K.; Veith, G.; Zuber, G.; Knorre, W.A. High cell density fermentation of recombinant Escherichia coli expressing human interferon alpha 1. Appl. Microbiol. Biotechnol. 1990, 34, 77-82. [CrossRef]

3. Schweder, T.; Krüger, E.; Xu, B.; Jürgen, B.; Blomsten, G.; Enfors, S.O.; Hecker, M. Monitoring of genes that respond to process-related stress in large-scale bioprocesses. Biotechnol. Bioeng. 1999, 65, 151-159. [CrossRef]

4. Hewitt, C.J.; Nienow, A.W. The scale-up of microbial batch and fed-batch fermentation processes. Adv. Appl. Microbiol. 2007, 62, 105-135.

5. Enfors, S.O.; Jahic, M.; Rozkov, A.; Xu, B.; Hecker, M.; Jürgen, B.; Krüger, E.; Schweder, T.; Hamer, G.; O’Beirne, D.; et al. Physiological responses to mixing in large-scale bioreactors. J. Biotechnol. 2001, 85, 175-185. [CrossRef]

6. Soini, J.; Falschlehner, C.; Liedert, C.; Bernhardt, J.; Vuoristo, J.; Neubauer, P. Norvaline is accumulated after a down-shift of oxygen in Escherichia coli W3110. Microb. Cell Fact. 2008, 7, 1-14. [CrossRef] [PubMed]

7. Wolfe, A.J. The acetate switch. Microbiol. Molec. Biol. Rev. 2005, 69, 12-50. [CrossRef]

8. Apostol, I.; Levine, J.; Lippincott, J.; Leach, J.; Hess, E.; Glascock, C.B.; Weickert, M.J.; Blackmore, R. Incorporation of norvaline at leucine positions in recombinant human hemoglobin expressed in Escherichia coli. J. Biol. Chem. 1997, 272, 28980-28988. [CrossRef] [PubMed]

9. Sycheva, E.V.; Yampol'skaya, T.A.; Preobrajenskaya, E.S.; Novikova, A.E.; Matrosov, N.G.; Stoynova, N.V. Overproduction of Noncanonical Amino Acids by Escherichia coli Cells. Microbiology 2007, 76, 712-718. [CrossRef] 
10. Reitz, C.; Fan, Q.; Neubauer, P. Synthesis of non-canonical branched-chain amino acids in Escherichia coli and approaches to avoid their incorporation into recombinant proteins. Curr. Opin. Biotechnol. 2018, 53, 248-253. [CrossRef]

11. Neubauer, P.; Junne, S. Scale-up and scale-down methodologies for bioreactors. In Bioreactors: Design, Operation and Novel Applications, 1st ed.; Mandenius, C.-F., Ed.; Wiley-VCH Verlag GmbH \& Co. KGaA: Weinheim, Germany, 2016 ; pp. 323-354.

12. Soini, J.; Ukkonen, K.; Neubauer, P. Accumulation of amino acids deriving from pyruvate in Escherichia coli W3110 during fed-batch cultivation in a two-compartment scale-down bioreactor. Adv. Biosci. Biotechnol. 2011, 2, 336-339. [CrossRef]

13. Anane, E.; García, Á.C.; Haby, B.; Hans, S.; Krausch, N.; Krewinkel, M.; Hauptmann, P.; Neubauer, P.; Cruz Bournazou, M.N. A model-based framework for parallel scale-down fed-batch cultivations in mini-bioreactors for accelerated phenotyping. Biotechnol. Bioengin. 2019, 116, 2906-2918. [CrossRef] [PubMed]

14. Kreth, J.; Lengeler, J.W.; Jahreis, K. Characterization of pyruvate uptake in Escherichia coli K12. PLoS ONE 2013, 8, e67125.

15. Panula-Perälä, J.; Šiurkus, J.; Vasala, A.; Wilmanowski, R.; Casteleijn, M.G.; Neubauer, P. Enzyme controlled glucose auto-delivery system for high cell density cultivations in microplates and shake flasks. Microb. Cell Fact. 2008, 7, 1-12. [CrossRef] [PubMed]

16. Xu, B.; Jahic, M.; Blomsten, G.; Enfors, S.O. Glucose overflow metabolism and mixed-acid fermentation in aerobic large-scale fed-batch processes with Escherichia coli. Appl. Microbiol. Biotechnol. 1999, 51, 564-571. [CrossRef] [PubMed]

17. Soini, J.; Ukkonen, K.; Neubauer, P. High cell density media for Escherichia coli are generally designed for aerobic cultivationsconsequences for large-scale bioprocesses and shake flask cultures. Microb. Cell Fact. 2008, 7, 1-11. [CrossRef] [PubMed]

18. Anane, E.; Sawatzki, A.; Neubauer, P.; Cruz Bournazou, M.N. Modelling concentration gradients in fed-batch cultivations of E. coli-towards the flexible design of scale-down experiments. J. Chem. Technol. Biotechnol. 2019, 94, 516-526. [CrossRef]

19. Steinsiek, S.; Bettenbrock, K. Glucose transport in Escherichia coli mutant strains with defects in sugar transport systems. J. Bacteriol. 2012, 194, 5897-5908. [CrossRef]

20. Naider, F.; Bohak, Z.; Yariv, J. Reversible alkylation of a methionine residue near the active site of $\alpha$-galactosidase. Biochemistry 1972, 11, 3202-3208. [CrossRef]

21. Yuan, T.; Vogel, H.J. Substitution of the methionine residues of calmodulin with the unnatural amino acid analogs ethionine and norleucine: Biochemical and and spectroscopic studies. Prot. Sci. 1999, 8, 113-121. [CrossRef]

22. Harris, R.P.; Kilby, P.M. Amino acid mis-incorporation in recombinant biopharmaceutical products. Curr. Opin. Biotechnol. 2014, 30, 45-50. [CrossRef] [PubMed] 